Pacific Journal of 


\title{
ON EMBEDDING SEMIFLOWS INTO A RADIAL FLOW ON $l_{2}$
}

\author{
ROGER C. MCCANN
}

Let $\pi$ be a semiflow on a separable metric space $X$ such that the negative escape time function is lower semicontinuous and $x \rightarrow x \pi t$ is a one-to-one mapping for each $t \in R^{+}$. If $\pi$ has a globally uniformly asymptotically stable critical point, then $\pi$ can be embedded into a radial flow on $l_{2}$. This generalizes known results on embedding flows or semiflows into radial flows on $l_{2}$.

1. Introduction. In [3] L. Janos showed that a semiflow $\pi$ on a compact metric space $X$ satisfying

(i) $\cdot \pi t$ is one-to-one for every $t \in R^{+}$

(ii) there is a $p \in X$ such that $\cap\{X \pi t: t \geqq 0\}=\{p\}$ can be embedded into a radial flow on $l_{2}$. In [2] M. Edelstein generalized this result to

THEOREM I. Let $\pi$ be a semiflow on a separable metric space $X$ satisfying

(a) for each $t \in R^{+}, x \rightarrow x \pi t: X \rightarrow X$ is a homeomorphism, of $X$ onto a closed subset of $X$,

(b) there is a $p \in X$ such that for each neighborhood $U$ of $p$ there is a $T \in R^{+}$such that $X \pi t \subset U$ for all $t \geqq T$. Then $\pi$ can be embedded into a radial flow on $l_{2}$.

Evidently properties (a) and (b) generalize properties (i) and (ii) respectively. Note that property (b) imposes a type of compactness on the semiflow. For example, a radial flow on $l_{2}$ can be embedded into itself trivially, but such a flow does not have property (b).

In this paper we further generalize properties (a) and (b) to

(c) $x \rightarrow x \pi t$ is one-to-one for each $t \in R^{+}$,

(d) the negative escape time function is lower semicontinuous,

(e) $\pi$ has a globally uniformly asymptotically stable critical point $p$.

We will show (Corollary 8) that property (a) implies properties (c) and (d). Evidently property (b) implies property (e). Property (e) imposes a type of local compactness on the semiflow. Notice that a radial flow on $l_{2}$ does satisfy property (e).

The principal result of this paper, Theorem 7, generalizes every other result known to the author concerning embedding flows or semiflows into radial flows on $l_{2}$. 
2. Notation and definitions. Throughout this paper $R$ and $R^{+}$will denote the reals and nonnegative reals respectively. A flow on a topological space $X$ is a continuous mapping $\pi: X \times R \rightarrow X$ such that (where $x \pi t=\pi(x, t)) x \pi 0=x$ for all $x \in X$ and $(x \pi t) \pi s=$ $x \pi(t+s)$ for all $x \in X$ and $t, s \in R$. If $R$ is replaced by $R^{+}$in the previous sentence, then $\pi$ is called a semiflow. A point $p$ of $X$ is called a critical point of $\pi$ if $p \pi t=p$ for all $t \in R$ (or $t \in R^{+}$if $\pi$ is a semiflow). A compact subset $M$ of $X$ is said to be stable with respect to $\pi$ if for any neighborhood $U$ of $M$ there is a neighborhood $V$ of $M$ such that $V \pi R^{+} \subset U$. A compact subset $M$ of $X$ is said to be a global attractor if for any neighborhood $U$ of $M$ and any $x \in X$ there is a $d \in R^{+}$such that $x \pi[d, \infty) \subset U$. The compact set $M$ is called a global uniform attractor if it is a global attractor and if there is a neighborhood $U$ of $M$ such that for any neighborhood $V \subset U$ of $M$ there is a $c \in R^{+}$such that $U \pi[c, \infty) \subset V$. A stable global (uniform) attractor is said to be globally (uniformly) asymptotically stable.

A continuous function $L: X \rightarrow R^{+}$is called a Liapunov function for a compact subset $M$ of $X$ if $L(x \pi t)<L(x)$ for every $x \in X-M$ and $0<t, L(x \pi t) \rightarrow 0$ as $t \rightarrow \infty$ for every $x \in X$, and $L(x)=0$ if $x \in M$. Let $M$ be a compact asymptotically stable subset of $X$. A straightforward argument shows that if $x \in X-M$ and if $U$ is any neighborhood of $M$, then there is a neighborhood $V$ of $x$ and a $T>0$ such that $V \pi[T, \infty) \subset U$. With this observation the proof of the following theorem is essentially identical with that of Theorem 10 in [1].

TheOREM II. A compact subset $M$ of a metric space $X$ is globally asymptotically stable with respect to a semiflow $\pi$ if and only if there is Liapunov function for $M$.

Let $X$ and $Y$ be topological spaces on which are defined flows (semiflows) $\pi$ and $\rho$ respectively. We say that $\pi$ can be embedded into $\rho$ if there is a homeomorphism $h$ of $X$ onto a subset of $Y$ such that $h(x \pi t)=h(x) \rho t$ for every $x \in X$ and $t \in R\left(t \in R^{+}\right)$.

The set of all sequences $x=\left\{x_{1}, x_{2}, \cdots, x_{n}, \cdots\right\}$ of real numbers such that $\sum_{n=1}^{\infty} x_{n}^{2}$ converges is denoted by $l_{2}$. If addition and scalar multiplication are defined coordinatewise and if a norm is defined by $\|x\|=\left(\sum_{n=1}^{\infty} x_{n}^{2}\right)^{1 / 2}$, then $l_{2}$ is a real Banach space. A flow $\rho$ on $l_{2}$ is called a radial flow if there is a $c \in(0,1)$ such that $x \rho t=c^{t} x$ for every $(x, t) \in l_{2} \times R$.

Let $\pi$ be a semiflow on $X$. The function $\alpha: X \rightarrow[-\infty, 0]$ defined by $\alpha(x)=\inf \{-t$ : there exists $y \in X$ with $y \pi t=x\}$ is called the negative escape time function. Throughout this paper we shall 
assume that $\alpha$ is lower semicontinuous, i.e., $\alpha(x) \leqq \lim _{y \rightarrow x} \inf \alpha(y)$. It is an elementary exercise to show that $\alpha(x \pi t)=\alpha(x)-t$ for all $t \geqq 0$ and $x \in X$.

3. The embedding. Henceforth, $\pi$ shall denote a semiflow on a separable metric space $X$ satisfying

(1) $x \rightarrow x \pi t$ is one-to-one for each $t \in R^{+}$,

(2) the negative escape time function is lower semicontinuous,

(3) $\pi$ has a globally unformly asymptotically stable critical point $p$.

Also, $U$ shall denote a neighborhood of $p$ such that for any neighborhood $V \subset U$ of $p$, there is a $T>0$ such that $U \pi[T, \infty) \subset V$.

Let $t<0$ and $x \in X$. Since $\cdot \pi(-t)$ is one-to-one there is at most one $y \in X$ with $y \pi(-t)=x$. If such a $y$ exists then we shall denote this $y$ by $x \pi t$. It is a straightforward exercise to show that if $s, t \in R$ and $x \in X$, then $(x \pi t) \pi s=x \pi(t+s)$ whenever each side of the equality is defined. Suppose that $\left\{x_{i}\right\}$ and $\left\{t_{i}\right\}$ are sequences in $X$ and $R$ converging to $x \in X$ and $t \in R$ respectively. Using property 2 it is easy to show that if $x_{i} \pi t_{i}$ is defined for each $i$, then $x \pi t$ is defined and $x_{i} \pi t_{i} \rightarrow x \pi t$ as $i \rightarrow \infty$.

LemMA 1. If $x \pi(\alpha(x), 0] \subset U$, then $-\infty<\alpha(x)$.

Proof. Let $V \subset U$ be a neighborhood of $p$ such that $V \pi R^{+}=V$ and $x \notin V$. Then $x \pi(\alpha(x), 0] \cap V=\phi$. Let $T>0$ be such that $U \pi T \subset V$. Then $x \pi(\alpha(x)+T, \infty) \subset V$. In order that this be consistent with $x \pi(\alpha(x), 0] \cap V=\phi$, we must have $\alpha(x) \neq-\infty$.

LeMma 2. Let $\sigma$ be a semiflow on a metric space $Z$. If

(i) the negative escape time function $\gamma$ is lower semicontinuous,

(ii) each trajectory contains a start point, i.e., for each $x \in Z$ there is a $y \in Z$ such that $y \sigma(-\gamma(x))=x$, then $Z \pi t$ is a closed subset of $Z$ for each $t \geqq 0$.

Proof. Let $t \geqq 0$ and let $\left\{x_{i}\right\}$ be a sequence in $Z$ such that $x_{i} \sigma t \rightarrow y$ for some $y \in Z$. Then $\gamma\left(x_{i} \sigma t\right) \leqq-t$ for every $i$ so that $\gamma(y) \leqq-t$. By (ii) there is a $z \in Z$ such that $z \sigma(-\gamma(y))=y$. Then $y=(z \pi(-\gamma(y)-t)) \sigma t \in Z \sigma t$. It follows that $Z \sigma \mathrm{t}$ is a closed subset of $Z$.

Let $L$ be a Liapunov function for $p$ (Theorem II) and let $\lambda$ be any number in the range of $L$ such that $L^{-1}([0, \lambda]) \subset U$. Set 


$$
Y=\left\{x \in L^{-1}([0, \lambda]): \alpha(x) \leqq-1 \text { and } x \pi(-1, \infty) \subset L^{-1}([0, \lambda])\right\}
$$

and let $\sigma$ denote the semiflow obtained by restricting $\pi$ to $Y \times R^{+}$. Let $\beta$ denote the negative escape time function with respect to $\sigma$.

We will show that $\sigma$ satisfies the hypotheses of Theorem I. Hence, $\sigma$ can be embedded into a radial flow on $l_{2}$. We will then extend this embedding to an embedding of $\pi$ into a radial flow.

Lemma 3. For every $x \in Y$ there is a $y \in Y$ such that $y \sigma(-\beta(x))=x$.

Proof. There are two cases to consider: $x \pi(\alpha(x), \infty) \subset L^{-1}([0, \lambda))$ and $x \pi(\alpha(x), \infty) \cap L^{-1}(\lambda) \neq \phi$. In the latter case there is a unique $z \in x \pi(\alpha(x), \infty) \cap L^{-1}(\lambda)$ and a unique $t \in R$ such that $z \pi t=x$. Since $x \in Y$ we must have $1 \leqq t$. Then $\beta(x)=-t+1$. Set $y=z \pi 1$. Then $y \in Y$ and $y \sigma(-\beta(x))=y \pi(-\beta(x))=(z \pi 1) \pi(t-1)=z \pi t=x$. Now suppose $x \pi(\alpha(x), \infty) \subset L^{-1}([0, \lambda))$. Then $x \pi(\alpha(x), \infty) \subset U$ so that, by Lemma $1,-\infty<\alpha(x)$. Since $x \in Y$ we must have $\alpha(x) \leqq-1$. Let $y \in x \pi(\alpha(x), \infty)$ be such that $y \pi(-\alpha(x)-1)=x$. Since $\alpha(x)=$ $\alpha(y \pi(-\alpha(x)+1))=\alpha(y)+\alpha(x)+1$ we have $\alpha(y)=-1$. If $y=z \pi t$ for some $t>0$ then $-1=\alpha(y)=\alpha(z \pi t)=\alpha(z)-t$ so that $\alpha(z)=$ $t-1>-1$. It follows that $\beta(x)=\alpha(x)+1$ and that $y \sigma(-\beta(x))=x$. This completes the proof.

Lemma 4. Let $\left\{x_{i}\right\}$ be a sequence such that $x_{i} \rightarrow x$ for some $x \in X$. If there exists a $t \in R$ such that $x \pi t \in L^{-1}(\lambda)$, then either $t \leqq \lim$ inf $\alpha\left(x_{i}\right)$ or there are a subsequence $\left\{x_{j}\right\}$ of $\left\{x_{i}\right\}$ and a sequence $\left\{t_{j}\right\}$ in $R$ such that $x_{j} \pi t_{j} \in L^{-1}(\lambda)$. In the latter case $t_{j} \rightarrow t$.

Proof. Suppose $\lim \inf \alpha\left(x_{i}\right)<t$. Let $\left\{x_{j}\right\}$ be a subsequence of $\left\{x_{i}\right\}$ such that $\alpha\left(x_{j}\right) \rightarrow \lim \inf \alpha\left(x_{i}\right)$. For any $\delta \in\left(0, t-\lim \inf \alpha\left(x_{i}\right)\right)$ eventually $\alpha\left(x_{j}\right)<t-\delta$. Also $\alpha(x) \leqq t-\delta$ because $\alpha(x) \leqq \lim$ inf $\alpha\left(x_{i}\right)$. Since $L(x \pi(t-\delta))>L(x \pi t)=\lambda>L(x \pi(t+\delta))$ we have $L\left(x_{j} \pi(t-\delta)\right)>\lambda>L\left(x_{j} \pi(t+\delta)\right)$ eventually. Hence, there are $t_{j} \in$ $(t-\delta, t+\delta)$, eventually, such that $L\left(x_{j} \pi t_{j}\right)=\lambda$. Since $\delta$ can be chosen arbitrarily small we must have $t_{j} \rightarrow t$.

LEMMA 5. $\beta$ is lower semicontinuous.

Proof. Let $x \in Y$ and let $\left\{x_{i}\right\}$ be a sequence in $Y$ such that $x_{j} \rightarrow x$. Let $\left\{x_{j}\right\}$ be any subsequence of $\left\{x_{i}\right\}$ such that $\beta\left(x_{j}\right) \rightarrow \beta$ for some $\beta \in[-\infty, 0]$. There are two cases to consider: $x \pi t \in L^{-1}(\lambda)$ for some $t \in R$ and $x \pi(\alpha(x), \infty) \subset L^{-1}([0, \lambda))$. If $x \pi t \in L^{-1}(\lambda)$ for some $t$, then by Lemma 4 either $\alpha(x) \leqq t \leqq \lim \inf \alpha\left(x_{j}\right)$ or there are a subsequence $\left\{x_{k}\right\}$ of $\left\{x_{j}\right\}$ and a sequence $\left\{t_{k}\right\}$ in $R$ such that $t_{k} \rightarrow t$ and $x_{k} \pi t_{k} \in$ 
$L^{-1}(\lambda)$. If $t \leqq \lim \inf \alpha\left(x_{j}\right)$, then $\beta(x)=t+1$ and $\beta\left(x_{j}\right)=\alpha\left(x_{j}\right)+1$ so that $\beta(x) \leqq \lim \inf \beta\left(x_{j}\right)=\beta$. If there are a subsequence $\left\{x_{k}\right\}$ of $\left\{x_{j}\right\}$ and a sequence $\left\{t_{k}\right\}$ in $R$ such that $t_{k} \rightarrow t$ and $x_{k} \pi t_{k} \in L^{-1}(\lambda)$, then $\beta(x)=$ $t+1$ and $\beta\left(x_{k}\right)=t_{k}+1$. Then $\beta(x)=\lim \beta\left(x_{k}\right)=\beta$. Thus if $x \pi t \in L^{-1}(\lambda)$, then $\beta(x) \leqq \beta$. It follows that $\beta(x) \leqq \lim \inf \beta\left(x_{i}\right)$ whenever $x \pi t \epsilon$ $L^{-1}(\lambda)$ for some $t \in R$. Now suppose that $x \pi(\alpha(x), \infty) \subset L^{-1}([0, \lambda))$. Then $\beta(x)=\alpha(x)+1$. Again there are two cases to consider: $x_{i} \pi\left(\alpha\left(x_{i}\right), \infty\right) \subset L^{-1}([0, \lambda))$ for every $i$ and there exist a subsequence $\left\{x_{n}\right\}$ of $\left\{x_{i}\right\}$ and a sequence $\left\{s_{n}\right\}$ in $R$ such that $x_{n} \pi s_{n} \in L^{-1}(\lambda)$ for every $n$. In the former case we have $\beta\left(x_{i}\right)=\alpha\left(x_{i}\right)+1$ and $\beta(x) \leqq$ $\lim \inf \beta\left(x_{i}\right)$ since $\alpha$ is lower semicontinuous. In the latter case, let $V \subset U$ be a neighborhood of $p$ such that $x \notin \overline{V \pi R^{+}}$and let $T>0$ be such that $U \pi[T, \infty) \subset V$. Then $L^{-1}(\lambda) \pi[T, \infty) \subset V$ and we must have $s_{n} \in[0, T]$ for all $n$ sufficiently large. Let $s$ be any accumulation point of $\left\{s_{n}\right\}$ and let $\left\{s_{j}\right\}$ be a subsequence of $\left\{s_{n}\right\}$ such that $s_{j} \rightarrow s$. Then $x_{j} \pi s_{j} \in L^{-1}(\lambda)$ and $x_{j} \pi s_{j} \rightarrow x \pi s$. Hence, $x \pi s \in L^{-1}(\lambda)$ which contradicts our assumption that $x \pi(\alpha(x), \infty) \subset L^{-1}([0, \lambda))$. It follows that $\beta(x) \leqq \lim \inf \beta\left(x_{i}\right)$ whenever $x \pi(\alpha(x), \infty) \subset L^{-1}([0, \lambda))$. Combining this with the result $\beta(x) \leqq \lim \inf \beta\left(x_{i}\right)$ whenever $x \pi t \epsilon$ $L^{-1}(\lambda)$ for some $t \in R$ obtained earlier in the proof, we conclude that $\beta$ is lower semicontinuous.

Collecting together the above results we have that

(i) $\sigma$ is a semiflow on the separable metric space $Y$,

(ii) if $V$ is a neighborhood in $Y$ of $p$, then there is a $T>0$ such that $Y \sigma[T, \infty) \subset V$, (This follows directly from the facts that $Y \subset U$ and $\sigma$ is a restriction of $\pi$.)

(iii) $Y \sigma t$ is a closed subset of $Y$ for every $t \geqq 0$ (Lemmas 3,5, and 2).

In light of Theorem I the semiflow $\sigma$ on $Y$ can be embedded into a radial flow $\rho$ on $l_{2}$. Let $c \in(0,1)$ be such that $x \rho t=c^{t} x$ and let $h: Y \rightarrow l_{2}$ be a homeomorphism of $Y$ onto $h(Y)$ such that $h(x \sigma t)=$ $h(x) \rho t$ for every $(x, t) \in Y \times R^{+}$. Since $\sigma$ is a restriction of $\pi$ we have $h(x \pi t)=h(x) \rho t$ for every $(x, t) \in Y \times R^{+}$. Now define a mapping $H: X \rightarrow l_{2}$ by

$$
H(x)=h(x \pi t) \rho(-t)
$$

where $t \in R^{+}$is such that $x \pi t \in Y$. ( $H$ will be shown to be well defined in the following lemma.)

Lemma $6 . \quad H$ is a homeomorphism of $X$ onto $H(X)$.

Proof. We will first show that $H$ is well defined. Clearly for 
every $x \in X$, there is a $t \geqq 0$ such that $x \pi t \in Y$. Moreover, if $x \pi t \epsilon$ $Y$, then $x \pi(t+s) \in Y$ for every $s \geqq 0$. In order to show that $H$ is well defined it suffices to show that $h(x \pi t) \rho(-t)=h(x \pi(t+s)) \rho(-t-s)$ whenever $x \pi t \in Y$ and $s \geqq 0$. Since $x \pi t \in Y$ and $s \geqq 0$ we have $h(x \pi(t+s))=h((x \pi t) \pi s)=h(x \pi t) \rho s$. Hence $h(x \pi(t+s)) \rho(-t-s)=$ $(h(x \pi t) \rho s) \rho(-t-s)=h(x \pi t) \rho(-t)$. The mapping $H$ is well defined. We will now show that $H$ is one-to-one. Suppose that $H(x)=$ $h(x \pi t) \rho(-t), H(y)=h(y \pi s) \rho(-s)$, and $H(x)=H(y)$. Without loss of generality we may assume that $t \geqq s$. Then $H(y)=h(y \pi t) \rho(-t)$ since $y \pi t \in Y$ whenever $y \pi s \in Y$ and $s \leqq t$. Since $H(x)=H(y)$ we must have $h(x \pi t)=h(y \pi t)$. Recalling that $h$ is a homeomorphism we have $x \pi t=y \pi t$ so that $x=y$ since $\cdot \pi t$ is one-to-one. The mapping $H$ is one-to-one. Next we will show that $H$ is continuous. Let $x \in X$ and let $\left\{x_{i}\right\}$ be a sequence in $X$ such that $x_{i} \rightarrow x$. Let $t \in R^{+}$be such that $L(x \pi t)<\lambda$. Then $x \pi(t+1) \in Y$. Also for all $i$ sufficiently large $L\left(x_{i} \pi t\right)<\lambda$ and $x_{\imath} \pi(t+1) \in Y$. Then $H\left(x_{i}\right)=$ $h\left(x_{i} \pi(t+1)\right) \rho(-t-1) \rightarrow h(x \pi(t+1)) \rho(-t-1)=H(x)$. Hence, $H$ is continuous. Finally we will prove that $H^{-1}$ is continuous. Let $y \in X$ and let $\left\{y_{i}\right\}$ be a sequence in $X$ such that $H\left(y_{i}\right) \rightarrow H(y)$. Then there exist $t, t_{i} \in R^{+}$such that $H\left(y_{i}\right)=h\left(y_{i} \pi t_{i}\right) \rho\left(-t_{i}\right)$ and $H(y)=h(y \pi t)$. Let $s_{i}=\inf \left\{s \in R^{+}: y_{i} \pi s \in Y\right\}$. We will show that $\left\{s_{i}\right\}$ is bounded. Suppose not. Then there is a subsequence $\left\{s_{j}\right\}$ of $\left\{s_{i}\right\}$ such that $s_{j} \rightarrow \infty$. If $y_{i} \in L^{-1}([0, \lambda])$, then $s_{i} \leqq 1$. Hence, we may assume $1 \leqq s_{j}$ and $y_{j} \notin L^{-1}([0, \lambda])$ for every $j$. Then $y_{j} \pi\left(s_{j}-1\right) \in L^{-1}(\lambda)$. Note that $H(y) \leftarrow H\left(y_{j}\right)=h\left(y_{j} \pi s_{j}\right) \rho\left(-s_{j}\right)=c^{-s_{j}} h\left(y_{j} \pi s_{j}\right)$. Since $s_{j} \rightarrow \infty$ and $c \in$ $(0,1)$ we have $c^{-s_{j}} \rightarrow \infty$. In order that $c^{-s_{j}} h\left(y_{j} \pi s_{j}\right) \rightarrow H(y)$ we must also have $h\left(y_{j} \pi s_{j}\right) \rightarrow \overline{0}$ where $\overline{0}$ is the origin in $l_{2}$. Since $h$ is a homeomorphism $y_{j} \pi s_{j} \rightarrow p$ so that $y_{j} \pi\left(s_{j}-1\right) \rightarrow p$. This is impossible because $y_{j} \pi\left(s_{j}-1\right) \in L^{-1}(\lambda)$ and $L(p)=0$. Hence $\left\{s_{i}\right\}$ must be bounded. Without loss of generality we may suppose that $0 \leqq s_{i} \leqq t$ for every $i$. Then $H\left(y_{i}\right)=h\left(y_{i} \pi t\right) \rho(-t) \rightarrow h(y \pi t) \rho(-t)=H(y)$ so that $h\left(y_{i} \pi t\right) \rightarrow$ $h(y \pi t)$. Since $h$ is a homeomorphism, $y_{i} \pi t \rightarrow y \pi t$ and we have $y_{i} \rightarrow y$. Hence, $H^{-1}$ is continuous and $H$ is a homeomorphism of $X$ onto $H(X) \subset l_{2}$.

THEOREM 7. Let $\pi$ be a semiflow on a separable metric space $X$ such that the negative escape time function is lower semicontinuous and $\pi t$ is one-to-one for each $t \in R^{+}$. If $\pi$ has a globally uniformly asymptotically stable critical point, then $\pi$ can be embedded into a radial flow on $l_{2}$.

Proof. In light of Lemma 6, we need only show that $H(x \pi s)=$ $H(x) \rho s$ for every $(x, s) \in X \times R^{+}$. Let $x \in X$ and $t \geqq 0$ be such that $x \pi t \in Y$. Then $(x \pi s) \pi t=x \pi(t+s) \in Y$ and we have $H(x \pi s)=$ 
$h((x \pi s) \pi t) \rho(-t)=h((x \pi t) \pi s) \rho(-t)=(h(x \pi t) \rho s) \rho(-t)=(h(x \pi t) \rho(-t)) \rho s=$ $H(x) \rho s$.

COROLlaRy 8. ([2, Theorem I].) Let $\pi$ be a semiflow on a separable metric space having the properties

(i) $x \rightarrow x \pi t$ is a homeomorphism of $X$ onto a closed subset of $X$ for each $t \in R^{+}$,

(ii) there is a $p \in X$ such that for any neighborhood $U$ of $p$ there is a $T \in R^{+}$with $X \pi t \subset U$ for all $t \geqq T$. Then $\pi$ can be embedded into a radial flow on $l_{2}$.

Proof. Clearly (i) and (ii) imply that $\cdot \pi t$ is one-to-one for all $t \in R^{+}$and $p$ is globally uniformly asymptotically stable respectively. It remains to show that (i) implies that the negative escape time function $\alpha$ is lower semicontinuous. Suppose that $\alpha$ is not lower semicontinuous. Then there exist $x \in X, \delta>0$, and a sequence $\left\{x_{i}\right\}$ in $X$ such that $x_{i} \rightarrow x$ and $\alpha\left(x_{i}\right)<\alpha(x)-\delta$ for every $i$. Thus $x_{\imath} \pi(\alpha(x)-\delta)$ is defined for every $i$. Then $\left(x_{i} \pi(\alpha(x)-\delta)\right) \pi(-\alpha(x)+\delta)=$ $x_{i} \rightarrow x$ so that $\left.x \in \overline{X \pi(-\alpha(x)+\delta}\right)=X \pi(-\alpha(x)+\delta)$ since $X \pi t$ is closed for every $t \geqq 0$. Then there exists $z \in X$ such that $z \pi(\alpha(x)-\delta)=x$. This is impossible because $\alpha(x)-\delta<\alpha(x)$ and $\alpha(x)=\inf \{-t$ : there exists $y \in X$ with $y \pi t=x\}$. Therefore, we must have that $\alpha$ is lower semicontinuous. The desired result now follows from Theorem 7 .

In the proof of Corollary 8 we showed that if $X \pi t$ is a closed subset of $X$ for all $t \in R^{+}$then the negative escape time function $\alpha$ is lower continuous. The converse of this is not valid. Let $X=$ $[0,1)$ and define $\pi: X \times R \rightarrow X$ by $x \pi t=e^{-t} x$. Evidently $\pi$ is a semiflow on $X$. The negative escape time function is defined by

$$
\alpha(x)= \begin{cases}\ln x & \text { if } x \neq 0 \\ -\infty & \text { if } x=0\end{cases}
$$

Thus $\alpha$ is lower semicontinuous. However $X \pi 1=\left[0, e^{-1}\right)$ is not a closed subset of $[0,1)$. Thus the lower semicontinuity of $\alpha$ does not imply that $X \pi t$ is a closed subset of $X$ for every $t \in R^{+}$.

Corollary 9. (Theorem 5 of [4].) Let $\pi$ be a flow on a separable metric space which has a globally asymptotically stable critical point p. Then $\pi$ can be embedded into a radial flow on $l_{2}$ if and only if $p$ is globally uniformly asymptotically stable.

Proof. Since $\pi$ is a flow $x \rightarrow x \pi t$ is one-to-one for every $t \in R^{+}$ and $\alpha(x)=-\infty$ for every $x \in X$. If $p$ is globally uniformly asymp- 
totically stable, then, by Theorem $7, \pi$ can be embedded into a radial flow on $\ell_{2}$. The converse follows easily since the origin in $\ell_{2}$ is globally uniformly asymptotically stable with respect to a radial flow.

\section{REFERENCES}

1. J. Auslander and P. Seibert, Prolongations and stability in dynamical systems, Ann. Inst. Fourier, Grenoble, 14 (1964), 237-268.

2. M. Edelstein, On the homomorphic and isomorphic embeddings of a semiflow into a radial flow, Pacific J. Math., 91 (1980), 281-291.

3. L. Janos, $A$ linearization of semiflows in the Hilbert space $l_{2}$, in Topology Proceedings (Baton Rouge, La.), 2 (1977), 219-232.

4. R. McCann, Embedding asymptotically stable dynamical systems into radial flows in $l_{2}$, Pacific J. Math., 90 (1980), 425-429.

Received June 12, 1980 and in revised form September 2, 1980.

MISSISSIPPI STATE UNIVERSITY

MississtPpi State, MS 39762 


\section{PACIFIC JOURNAL OF MATHEMATICS}

\section{EDITORS}

DONALD BABBITT (Managing Editor)

University of California

Los Angeles, California 90024

Hugo RossI

University of Utah

Salt Lake City, UT 84112

C. C. MOORE and ANDREW OGG

University of California

Berkeley, CA 94720

\section{J. DugundJI}

Department of Mathematics University of Southern California Los Angeles, California 90007

R. Finn and J. Milgram Stanford University

Stanford, California 94305

\section{ASSOCIATE EDITORS}
R. ARENS
E. F. BeCKENBACH
B. H. Neumann
F. WoLF
K. YoSHIDA

\section{SUPPORTING INSTITUTIONS}

UNIVERSITY OF ARIZONA

UNIVERSITY OF BRITISH COLUMBIA

CALIFORNIA INSTITUTE OF TECHNOLOGY

UNIVERSITY OF CALIFORNIA

MONTANA STATE UNIVERSITY

UNIVERSITY OF NEVADA, RENO

NEW MEXICO STATE UNIVERSITY

OREGON STATE UNIVERSITY
UNIVERSITY OF OREGON

UNIVERSITY OF SOUTHERN CALIFORNIA

STANFORD UNIVERSITY

UNIVERSITY OF HAWAII

UNIVERSITY OF TOKYO

UNIVERSITY OF UTAH

WASHINGTON STATE UNIVERSITY

UNIVERSITY OF WASHINGTON 


\section{Pacific Journal of Mathematics}

\section{Vol. 97, No. $1 \quad$ January, 1981}

Charles A. Asmuth and Joe Repka, Tensor products for $S L_{2}(\mathfrak{k})$. II.

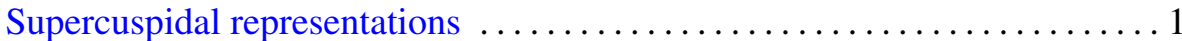

Joseph Barback, On finite sums of regressive isols . ................. 19

Matthew G. Brin and Daniel Russell McMillan, Jr., Generalized

three-manifolds with zero-dimensional nonmanifold set ............29

Kun Soo Chang, Converse measurability theorems for Yeh-Wiener space . . . 59

Christopher Brian Croke, A "maximal torus" type theorem for complete

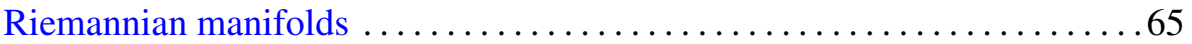

Gustave Adam Efroymson, Sums of squares in planar Nash rings . . . . . . 75

John Robert Fisher, Axiomatic radical and semisimple classes of rings . . . .81

Betty Kvarda, Consecutive integers for which $n^{2}+1$ is composite .......93

Roosevelt Gentry, New diagram proofs of the Hausdorff-Young theorem

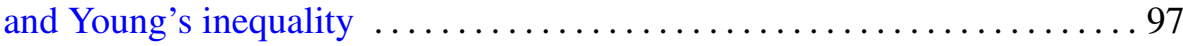

Patrick M. Gilmer, Topological proof of the $G$-signature theorem for $G$

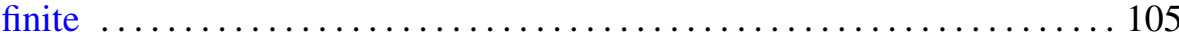

Chung Wei Ha, A noncompact minimax theorem .................. 115

James J. Hebda, Manifolds admitting taut hyperspheres ................ 119

Takayuki Kawada, Sample functions of Pólya processes ............. 125

Peter K. F. Kuhfittig, Common fixed points of nonexpansive mappings by

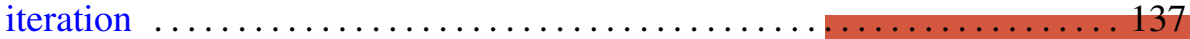

James Thomas Loats and Judith Roitman, Almost rigid Hopfian and dual

Hopfian atomic Boolean algebras .......................... 141

Roger McCann, On embedding semiflows into a radial flow on $l_{2} \ldots \ldots \ldots 151$

John McDonald, Closed orbits of convex sets of operators on the disk

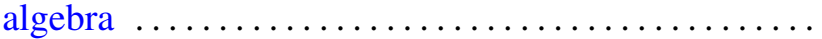

Mark D. Meyerson, Convexity and the table theorem .............. 167

Arnold William Miller, Generic Souslin sets . . . . . . . . . . . . . . . . 171

Takemi Mizokami, On the closed images of paracomplexes $\ldots \ldots \ldots \ldots \ldots 3$

Jagannadham Venkata Pakala and Thomas Stephen Shores, On

compactly packed rings $\ldots \ldots \ldots \ldots \ldots \ldots \ldots \ldots \ldots$

Andrew Pletch, Strong completeness in profinite groups

Wilbur Carrington Whitten, Inverting double knots

James Juei-Chin Yeh, Existence of strong solutions for stochastic

differential equations in the plane 